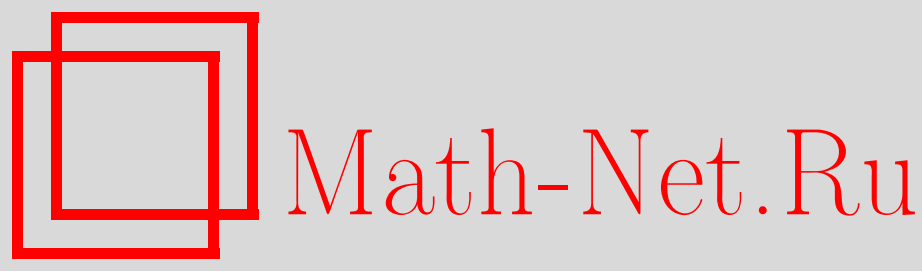

В. А. Андреев, Д. М. Давидович, Л. Д. Давидович, М. Д. Давидович, В. И. Манько, М. А. Манько, Трансформационное свойство функции Хусими и ее связь с функцией Вигнера и симплектическими томограммами, ТМФ, 2011, том 166, номер 3, 410-424

DOI: https://doi.org/10.4213/tmf6619

Использование Общероссийского математического портала Math-Net.Ru подразумевает, что вы прочитали и согласны с пользовательским соглашением http: //www . mathnet.ru/rus/agreement

Параметры загрузки:

IP: 54.157 .27 .8

26 апреля 2023 г., 16:53:24

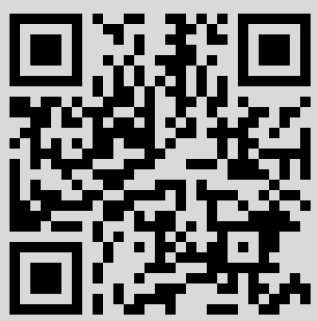




\title{
ТРАНСФОРМАЦИОННОЕ СВОЙСТВО ФУНКЦИИ ХУСИМИ И ЕЕ СВЯЗЬ С ФУНКЦИЕЙ ВИГНЕРА И СИМПЛЕКТИЧЕСКИМИ ТОМОГРАММАМИ
}

\begin{abstract}
Рассматриваются $Q$-функции Хусими, являющиеся квантовыми функциями распределения квазивероятностей на фазовом пространстве. Изучаются их трансформационные свойства при масштабном преобразовании $(q, p) \rightarrow(\lambda q, \lambda p)$. Доказана теорема, согласно которой при таком преобразовании функция Хусими физического состояния переходит в функцию, также являющуюся функцией Хусими некоторого физического состояния. Таким образом, это преобразование задает положительное отображение оператора плотности. Изучена связь функций Хусими с функциями Вигнера и симплектическими томограммами. Установлено, как они преобразуются при масштабном преобразовании. В качестве примера рассмотрен гармонический осциллятор. Показано, как его состояния преобразуются при масштабном преобразовании.
\end{abstract}

Ключевые слова: квантовая механика, функции Хусими, функции Вигнера, симплектические томограммы, масштабное преобразование.

\section{1. ВВЕДЕНИЕ}

Квантовая механика является статистической теорией, результат измерения в ней можно предсказать, как правило, только с некоторой вероятностью, и в этом она похожа на классические статистические теории. Основным объектом в классической статистической теории является функция распределения $\rho(q, p)$ - плотность вероятности того, что система находится в состоянии, характеризующемся параметрами $(q, p)$. Она определена на фазовом пространстве системы, и ее аргументы имеют смысл координаты и импульса. При этом предполагается, что координаты

*Физический институт им. П. Н. Лебедева РАН, Москва, Россия.

E-mail: andrvlad@yandex.ru

${ }^{\dagger}$ Institute of Nuclear Sciences Vinca, Belgrade, Serbia. E-mail: davidd@vinca.rs

${ }^{\ddagger}$ Institute of Physics, Belgrade, Serbia. E-mail: Ljubica.davidovich@gmail.com

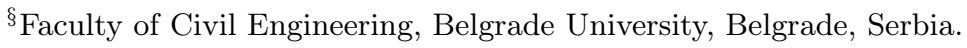

E-mail: davidovich.Milena@gmail.com 
и импульсы можно измерить совместно. Зная функцию распределения системы, можно вычислить различные характеристики, например средние значения тех или иных величин.

Поскольку квантовая механика также является статистической теорией, с самого начала ее развития предпринимались попытки описать квантовые состояния так же, как они описываются в классической статистической механике. Понятно, что речь может идти только о каком-то аналоге классической функции распределения, поскольку в силу принципа неопределенности квантовые состояния не могут одновременно обладать определенными значениями координаты и импульса.

В 1932 году была введена функция Вигнера $W(q, p)$ [1]. Она определяется как преобразование Вейля ядра оператора плотности в координатном представлении. Поскольку оператор плотности эрмитов, функция Вигнера действительна. Это преобразование обратимо, и по функции Вигнера можно восстановить матрицу плотности состояния. Таким образом, функция $W(q, p)$ полностью описывает квантовое состояние. Интеграл от функции Вигнера по импульсу равен плотности вероятности координаты, а интеграл по координате - плотности вероятности импульса. Точно такими же свойствами обладает функция распределения в классической статистической механике. Функция $W(q, p)$ является распределением в фазовом пространстве, однако ее нельзя интерпретировать как некоторую плотность вероятности, в первую очередь потому, что $W(q, p)$ может принимать отрицательные значения. Поэтому функцию Вигнера принято называть распределением квазивероятностей. Ее свойства подробно исследованы в обзорах [2], [3].

Существуют и другие квантовые функции распределения на фазовом пространстве. Наиболее известны $Q$-функция Хусими-Кано [4], [5] (мы будем называть ее функцией Хусими) и $P$-функция Глаубера-Сударшана [6], [7].

Общая проблематика представления квантовой механики в фазовом пространстве обсуждалась в работах [8]. Свойства квантовых функций распределения рассматривались в книге [9]; в частности, было показано, что функция Хусими является плотностью совместного распределения коммутирующих наблюдаемых $q-q_{0}, p-p_{0}$, где $q_{0}, p_{0}$ - вспомогательная мода в вакуумном состоянии, и, таким образом, реализуется одним измерением в одном статистическом эксперименте. Однако подчеркнем, хотя это и очевидно, что в силу принципа неопределенности функцию Хусими нельзя интерпретировать как совместное распределение вероятностей координаты $q$ и импульса $p$ некоторой квантовой системы. Другие аспекты квантовых распределений, связанных, в частности, с $P$-функцией Глаубера-Сударшана, рассматривались в работе [10].

Паули поставил вопрос о возможности полного описания квантового состояния путем задания двух распределений вероятностей - распределения по координате и распределения по импульсам [11]. Хотя эта задача имеет отрицательный ответ, при ее рассмотрении были построены схемы квантовой томографии [12]. А именно, была введена функция $w(X, \mu, \nu)$, связанная взаимно однозначным преобразованием с функцией Вигнера, которая имеет смысл плотности вероятности величины $X=\mu q+\nu p$. Величину $X$ можно понимать как координату в повернутой системе отсчета в фазовом пространстве $(q, p)$. Функция $w(X, \mu, \nu)$ полностью определяет квантовое состояние. Ее называют симплектической томограммой квантового состояния. Свойства и значение томограмм обсуждались в обзоре [13]. Набор величин 
$w(X, \mu, \nu)$ является континуальным семейством плотностей распределений вероятностей, зависящих от параметров $\mu, \nu$. Это соответствует тому, что семейство томограмм отвечает измерениям континуального набора попарно некоммутирующих наблюдаемых, и поэтому в принципе предполагает не одно измерение, а множество несовместимых измерений для различных $\mu, \nu$. В квантовой оптике каждое такое измерение фиксируется фазой $\theta$ локального осциллятора. Множество измерений, сделанных при достаточном количестве разных углов $\theta$ таких, что $\mu=\cos \theta$, $\nu=\sin \theta$, позволяет восстановить оператор плотности квантовой системы [14]. Таким образом, на основе экспериментально полученных томограмм $w(X, \mu, \nu)$ можно рассчитать функции Вигнера, Хусими и Глаубера-Сударшана. Задача Паули также обсуждалась в работе [15].

Функции Вигнера и Хусими широко используются при исследовании конкретных физических задач, большой интерес вызывают их аналитические свойства и связи друг с другом и симплектическими томограммами. Квантовую механику можно сформулировать как в терминах функции Вигнера, так и в терминах функции Хусими [16], [17]. Укажем на некоторые физические задачи, в которых применяется функция Хусими $Q(q, p)$.

Одной из характеристик функции Хусими, часто использующейся в прикладных задачах, например при изучении квантового хаоса и свойств квантовых бильярдов [18], [19], является число и локализация ее нулей. Топология распределения нулей функции Хусими применялась в работе [20] для изучения колебательной динамики сложных молекулярных систем. В общем виде связь между структурой нулей функции Хусими и физическими свойствами состояний рассматривалась в работе [21]. Также связь между аналитическими свойствами функции Хусими и содержащейся в ней информацией изучалась в работе [22]. В работе [23] с помощью функций Вигнера и Хусими были исследованы свойства когерентных состояний ангармонического осциллятора, показано, что эти состояния имеют субпуассонову статистику.

Также функцию Хусими можно использовать при вычислении различных вероятностей. В работе [24] для произвольного нестационарного $N$-мерного гамильтониана была построена многомерная функция Хусими и показано, что она является производящей функцией вероятностей перехода между фоковскими состояниями. В работах [25], [26] функция Хусими использовалась для анализа свойств зацепленности в различных сложных системах. В работах [27], [28] исследовалась связь функции Хусими с соотношениями неопределенности.

В настоящей работе изучаются связи между функциями Вигнера, Хусими и томограммами. Найдены интегральные и дифференциальные соотношения, выражающие их друг через друга. В разделе 2 приведены определение и основные свойства функции Хусими. В разделе 3 найдены соотношения, связывающие функцию Вигнера и функцию Хусими, а в разделе 4 - соотношения, связывающие функцию Хусими и томограмму. В разделе 5 исследуется поведение функций Хусими и Вигнера при масштабном преобразовании

$$
(q, p) \rightarrow(\lambda q, \lambda p)
$$

Известно, что при таком преобразовании функция Хусими $Q(q, p)$ переходит в $\lambda^{2} Q(\lambda q, \lambda p)$, которая также является функцией Хусими некоторого физического состояния, если $\lambda$ - вещественное число и $|\lambda| \leqslant 1$. Данное утверждение было 
высказано в работе [29]. В качестве его обоснования были использованы некоторые общие свойства функций Хусими. В настоящей работе мы даем совершенно другое, чисто аналитическое доказательство этого факта. Оно обладает также тем достоинством, что в процессе доказательства возникают некоторые конструкции, которые можно использовать и для других целей. В частности, получено явное выражение для ядра матрицы плотности преобразованного состояния. Это доказательство содержится в приложении. Также в разделе 5 найдено преобразование, отвечающее масштабному преобразованию (1) и переводящее функцию Вигнера в функцию Вигнера. В разделе 6 в качестве примера рассмотрен гармонический осциллятор. Показано, каким именно физическим состояниям отвечает преобразованная функция Хусими $\lambda^{2} Q(\lambda q, \lambda p)$, если исходная величина $Q(q, p)$ является функцией Хусими чистого фоковского состояния.

Заметим, что преобразование (1) естественным образом возникает в ряде физических задач, например в задаче о квантовом усилителе [30]. При этом параметр $\lambda$ равен обратной величине коэффициента усиления, $\lambda=G^{-1}$.

\section{2. ОПРЕДЕЛЕНИЕ ФУНКЦИИ ХУСИМИ И ЕЕ СВОЙСТВА}

Функция Хусими определяется через оператор плотности состояния и набор когерентных состояний гармонического осциллятора [4], [5]. Пусть имеется некоторое состояние, которое задается оператором плотности $\hat{\rho}$, и когерентное состояние $\langle x \mid \alpha\rangle$. Тогда функцией Хусими состояния $\hat{\rho}$ называется функция

$$
Q\left(\alpha, \alpha^{*}\right)=\frac{1}{\pi} \int\langle\alpha \mid x\rangle \rho(x, y)\langle y \mid \alpha\rangle d x d y .
$$

Здесь $\alpha=\alpha_{\mathrm{r}}+i \alpha_{\mathrm{i}}-$ произвольное комплексное число, $\rho(x, y)$ - ядро оператора плотности в координатном представлении, а когерентное состояние $\langle x \mid \alpha\rangle$ имеет вид

$$
\langle x \mid \alpha\rangle=\left(\frac{1}{\pi a}\right)^{1 / 4} \exp \left[-\frac{1}{2}(x-\sqrt{2} \alpha)^{2}+i \alpha \alpha_{i}\right],
$$

мы полагаем $\hbar=1$. Функция Хусими неотрицательна всюду в фазовом пространстве, удовлетворяет неравенству $0 \leqslant Q\left(\alpha, \alpha^{*}\right) \leqslant 1 / \pi$ и нормирована на единицу,

$$
\int Q\left(\alpha, \alpha^{*}\right) d^{2} \alpha=1
$$

Для комплексного числа $\alpha$, задающего когерентное состояние, мы часто будем использовать представление $\alpha=(q+i p) / \sqrt{2}$ и рассматривать функцию Хусими $Q$ как функцию аргументов $p$ и $q$ :

$$
Q(q, p)=\frac{1}{2 \pi}\langle q, p|\hat{\rho}| q, p\rangle,
$$

где $|q, p\rangle$ - когерентное состояние, выраженное в переменных $q, p$.

Представляет интерес вопрос о том, какими свойствами должна обладать функция $F(q, p)$ двух переменных в фазовом пространстве, чтобы она являлась функцией Хусими некоторого физического состояния. Данная проблема обсуждалась в работе [31]. Другой подход к проблеме того, является ли данная функция $F(q, p)$ 
функцией Хусими, основан на использовании ее определения (2), точнее говоря, на том, как она выражается через когерентные состояния и оператор плотности. Используя явный вид когерентных состояний и свойства оператора плотности, можно установить, является ли рассматриваемая функция функцией Хусими. Ниже мы продемонстрируем эффективность такого подхода на конкретном примере.

$\mathrm{C}$ помощью функции Хусими можно вычислить средние значения антинормально упорядоченных произведений операторов рождения и уничтожения.

В настоящей работе мы изучаем свойства функции Хусими при некоторых преобразованиях координат, а также ее связь с функцией Вигнера и томограммами.

\section{3. СВЯЗЬ ФУНКЦИИ ВИГНЕРА С ФУНКЦИЕЙ ХУСИМИ}

Функция Вигнера $W(q, p)$ выражается через оператор плотности $\hat{\rho}$ следующим образом:

$$
W(q, p)=\int \rho\left(q+\frac{u}{2}, q-\frac{u}{2}\right) e^{-i p u} d u .
$$

Выражение для функции Хусими через функцию Вигнера $W(q, p)$ приведено в работах [32], [33]. Оно имеет вид

$$
Q(q, p)=\exp \left(\frac{1}{4} \frac{\partial^{2}}{\partial q^{2}}+\frac{1}{4} \frac{\partial^{2}}{\partial p^{2}}\right) W(q, p) .
$$

Данное равенство можно обратить и получить, как функция Вигнера выражается через функцию Хусими [31]:

$$
W(q, p)=\exp \left(-\frac{1}{4} \frac{\partial^{2}}{\partial q^{2}}-\frac{1}{4} \frac{\partial^{2}}{\partial p^{2}}\right) Q(q, p) .
$$

Используя преобразование Фурье, можно перейти от дифференциального соотношения (4) к его интегральному аналогу [2], [31]:

$$
Q(q, p)=\frac{1}{\pi} \int \exp \left[-\left(q-q^{\prime}\right)^{2}-\left(p-p^{\prime}\right)^{2}\right] W\left(q^{\prime}, p^{\prime}\right) d q^{\prime} d p^{\prime}
$$

В работах [34] приведено выражение для ядра оператора плотности через функцию Хусими:

$$
\begin{aligned}
\rho\left(x, x^{\prime}\right)= & \exp \left[\frac{1}{2}\left(x-x^{\prime}\right)^{2}\right] \sum_{n=0}^{\infty} \frac{(-1)^{n}}{2^{n} n !} \times \\
& \times \int H_{2 n}\left(q-\frac{1}{2}\left(x+x^{\prime}\right)^{2}\right) K\left(q, p ; x, x^{\prime}\right) Q(q, p) d q d p
\end{aligned}
$$

где $H_{2 n}(x)$ - полином Эрмита,

$$
K\left(q, p ; x, x^{\prime}\right)=\frac{1}{\sqrt{\pi}} \exp \left[-\left(q-\frac{1}{2}\left(x+x^{\prime}\right)\right)^{2}-\left(x-x^{\prime}\right)^{2}+i p\left(x-x^{\prime}\right)\right] .
$$

Более простая формула для ядра оператора плотности найдена в работе [31]:

$$
\begin{aligned}
\rho(x, y)= & \frac{1}{2 \hbar} \sqrt{\frac{1}{\pi}} \exp \left[\frac{1}{2}\left(x^{2}+y^{2}\right)\right] \int Q\left(\frac{i}{2 \hbar}\left(p_{1}-p_{2}\right), \frac{1}{2}\left(p_{1}+p_{2}\right)\right) \times \\
& \times \exp \left[-\frac{1}{4}\left(p_{1}-p_{2}\right)^{2}-i p_{1} x+i p_{2} y\right] d p_{1} d p_{2} .
\end{aligned}
$$


Можно выразить обратно функцию Хусими через оператор плотности, если использовать формулы (4), (6) и определение функции Вигнера (3).

\section{4. СВЯЗЬ ТОМОГРАММ С ФУНКЦИЕЙ ХУСИМИ}

Симплектические томограммы были введены в работах [12]. Их можно определить через функцию Вигнера:

$$
w(X, \mu, \nu)=\frac{1}{(2 \pi)^{2}} \int W(q, p) e^{-i k(X-\mu q-\nu p)} d k d q d p .
$$

Обратное преобразование выглядит следующим образом:

$$
W(q, p)=\frac{1}{2 \pi} \int w(X, \mu, \nu) e^{-i(\mu q+\nu p-X)} d X d \mu d \nu .
$$

Симплектические томограммы можно также задавать, используя матрицу плотности состояния $\hat{\rho}[35]$ :

$$
w(X, \mu, \nu)=\langle X, \mu, \nu|\hat{\rho}| X, \mu, \nu\rangle .
$$

Здесь $|X, \mu, \nu\rangle$ - собственное состояние эрмитового оператора $\widehat{X}_{\mu \nu}$, отвечающее собственному значению $X$, а $\mu$ и $\nu$ - вещественные параметры.

С другой стороны, мы знаем формулу (6), связывающую функцию Хусими с функцией Вигнера. Подставляя (10) в эту формулу, получаем выражение функции Хусими через томограмму:

$$
Q(q, p)=\frac{1}{2 \pi} \int w(X, \mu, \nu) e^{-\left(\mu^{2}+\nu^{2}\right) / 4} e^{-i(\mu q+\nu p-X)} d X d \mu d \nu .
$$

Используя равенство (5) для функции Вигнера и формулу (9), можно выразить томограмму через функцию Хусими:

$$
\begin{aligned}
w(X, \mu, \nu) & =\frac{1}{(2 \pi)^{2}} \int \exp \left(-\frac{1}{4} \frac{\partial^{2}}{\partial q^{2}}-\frac{1}{4} \frac{\partial^{2}}{\partial p^{2}}\right) Q(q, p) e^{-i k(X-\mu q-\nu p)} d k d q d p= \\
& =\frac{1}{(2 \pi)^{2}} \int \exp \left[\frac{k^{2}}{4}\left(\mu^{2}+\nu^{2}\right)\right] Q(q, p) e^{-i k(X-\mu q-\nu p)} d k d q d p .
\end{aligned}
$$

\section{5. ПРЕОБРАЗОВАНИЯ ФУНКЦИИ ХУСИМИ И ФУНКЦИИ ВИГНЕРА}

Рассмотрим преобразование функции Хусими следующего вида:

$$
Q_{\lambda}(q, p)=\lambda^{2} Q(\lambda q, \lambda p)
$$

Как уже отмечалось, оно возникает в ряде физических задач, например в работе [30] с его помощью описано действие квантового усилителя и показано, что функции Хусими состояний, входящих в усилитель и выходящих из него, связаны соотношением

$$
Q_{\text {out }}(\alpha)=\frac{1}{G^{2}} Q_{\text {in }}\left(\frac{\alpha}{G^{2}}\right)
$$


где $G$ - коэффициент усиления. В работе [29] показано, что если $\lambda^{2} \leqslant 1$ и $Q(q, p)-$ функция Хусими, то и $Q_{\lambda}(q, p)$ тоже является функцией Хусими некоторого физического состояния. В приложении к настоящей статье дано новое доказательство этого факта. Оно обладает тем достоинством, что позволяет найти явное выражение для матрицы плотности преобразованного состояния.

Известно также, что преобразование функции Вигнера, аналогичное преобразованию (13),

$$
\widetilde{W}^{\lambda}(q, p)=\lambda^{2} W(\lambda q, \lambda p),
$$

не является положительным и не переводит функцию Вигнера в функцию Вигнера. В работе [29] этот факт продемонстрирован на примере первого возбужденного состояния осциллятора. К функции Вигнера этого состояния применялось преобразование (14), а к получившейся функции $\widetilde{W}^{\lambda}(q, p)$ - преобразование (4), переводящее функцию Вигнера в функцию Хусими. Однако функция, получающаяся в результате, может принимать отрицательные значения, поэтому ее нельзя интерпретировать как функцию Хусими. Отсюда следует вывод, что и $\widetilde{W}^{\lambda}(q, p)$ не является функцией Вигнера какого-либо физического состояния. Тем не менее мы можем построить преобразование функции Вигнера, которое порождается преобразованием (1). Для этого следует сначала с помощью формул (4), (6) построить по исходной функции Вигнера отвечающую ей функцию Хусими, затем к этой функции Хусими применить преобразование (1), после чего с помощью формулы (5) построить новую функцию Вигнера. Это преобразование переводит функции Вигнера в функции Вигнера. Найдем его явный вид.

Функция Вигнера выражается через функцию Хусими по формуле (5). Для преобразованной функции Хусими из соотношения (13) мы имеем

$$
W^{\lambda}(q, p)=\exp \left(-\frac{1}{4} \frac{\partial^{2}}{\partial q^{2}}-\frac{1}{4} \frac{\partial^{2}}{\partial p^{2}}\right) \lambda^{2} Q(\lambda q, \lambda p) .
$$

Данное выражение можно привести к виду

$$
\begin{aligned}
W^{\lambda}(q, p)= & \exp \left(-\frac{1}{4} \frac{\partial^{2}}{\partial q^{2}}-\frac{1}{4} \frac{\partial^{2}}{\partial p^{2}}+\frac{1}{4 \lambda^{2}} \frac{\partial^{2}}{\partial q^{2}}+\frac{1}{4 \lambda^{2}} \frac{\partial^{2}}{\partial p^{2}}\right) \times \\
& \times \exp \left(-\frac{1}{4 \lambda^{2}} \frac{\partial^{2}}{\partial q^{2}}-\frac{1}{4 \lambda^{2}} \frac{\partial^{2}}{\partial p^{2}}\right) \lambda^{2} Q(\lambda q, \lambda p)= \\
& =\lambda^{2} \exp \left(-\frac{1}{4} \frac{\partial^{2}}{\partial q^{2}}-\frac{1}{4} \frac{\partial^{2}}{\partial p^{2}}+\frac{1}{4 \lambda^{2}} \frac{\partial^{2}}{\partial q^{2}}+\frac{1}{4 \lambda^{2}} \frac{\partial^{2}}{\partial p^{2}}\right) W(\lambda q, \lambda p),
\end{aligned}
$$

который показывает, как преобразуется функция Вигнера в том случае, когда функция Хусими преобразуется согласно правилу (13). Полученное выражение является дифференциальным и служит аналогом соотношений (4) и (5). Для функций Вигнера $W(q, p), W^{\lambda}(q, p)$ также можно найти связывающее их интегральное соотношение, аналогичное (6). Для этого следует воспользоваться преобразованием Фурье функции $W(\lambda q, \lambda p)$,

$$
W_{\mathrm{F}}(u, v)=\int e^{i\left(u q^{\prime}+v p^{\prime}\right)} W\left(\lambda q^{\prime}, \lambda p^{\prime}\right) d q^{\prime} d p^{\prime} .
$$


Выполняя обратное преобразование Фурье, получим тождество

$$
W(\lambda q, \lambda p)=\frac{1}{2 \pi^{2}} \int \exp \left[i\left(q^{\prime}-q\right) p^{\prime \prime}+i\left(p^{\prime}-p\right) q^{\prime \prime}\right] W\left(\lambda q^{\prime}, \lambda p^{\prime}\right) d q^{\prime \prime} d p^{\prime \prime} d q^{\prime} d p^{\prime}
$$

Подействуем на данную функцию оператором

$$
\lambda^{2} \exp \left[-\frac{1}{4}\left(1-\frac{1}{\lambda^{2}}\right) \frac{\partial^{2}}{\partial q^{2}}-\frac{1}{4}\left(1-\frac{1}{\lambda^{2}}\right) \frac{\partial^{2}}{\partial p^{2}}\right]
$$

получаем

$$
\begin{aligned}
W^{\lambda}(q, p)= & \frac{\lambda^{2}}{(2 \pi)^{2}} \int \exp \left[-\frac{1}{4}\left(1-\frac{1}{\lambda^{2}}\right)\left(i p^{\prime \prime}\right)^{2}-\frac{1}{4}\left(1-\frac{1}{\lambda^{2}}\right)\left(q^{\prime \prime}\right)^{2}\right] \times \\
& \times \exp \left[i\left(q-q^{\prime}\right) p^{\prime \prime}+i\left(p-p^{\prime}\right) q^{\prime \prime}\right] W\left(\lambda q^{\prime}, \lambda p^{\prime}\right) d q^{\prime \prime} d p^{\prime \prime} d q^{\prime} d p^{\prime} .
\end{aligned}
$$

Чтобы преобразовать это выражение, необходимо использовать следующие интегралы:

$$
\begin{gathered}
\int \exp \left[\frac{1}{4}\left(1-\frac{1}{\lambda^{2}}\right)\left(p^{\prime \prime}\right)^{2}+i\left(q-q^{\prime}\right) p^{\prime \prime}\right] d p^{\prime \prime}= \\
=2 \sqrt{\frac{\pi \lambda^{2}}{1-\lambda^{2}}} \exp \left[-\frac{\lambda^{2}\left(q^{\prime}-q\right)^{2}}{1-\lambda^{2}}\right], \\
\int \exp \left[\frac{1}{4}\left(1-\frac{1}{\lambda^{2}}\right)\left(q^{\prime \prime}\right)^{2}+i\left(p-p^{\prime}\right) q^{\prime \prime}\right] d q^{\prime \prime}= \\
=2 \sqrt{\pi} \sqrt{\frac{\lambda^{2}}{1-\lambda^{2}}} \exp \left[-\frac{\lambda^{2}\left(p^{\prime}-p\right)^{2}}{1-\lambda^{2}}\right] .
\end{gathered}
$$

Подставляя их в формулу (15), получаем

$$
\begin{aligned}
W^{\lambda}(q, p)= & \frac{1}{\pi} \frac{\lambda^{4}}{1-\lambda^{2}} \int \exp \left[-\frac{\lambda^{2}}{1-\lambda^{2}}\left(q^{\prime}-q\right)^{2}-\frac{\lambda^{2}}{1-\lambda^{2}}\left(p^{\prime}-p\right)^{2}\right] \times \\
& \times W\left(\lambda q^{\prime}, \lambda p^{\prime}\right) d q^{\prime} d p^{\prime} .
\end{aligned}
$$

Делая замену переменных $\lambda q^{\prime}=q^{\prime \prime}, \lambda p^{\prime}=p^{\prime \prime}$, получаем окончательный ответ,

$$
W^{\lambda}(q, p)=\frac{1}{\pi} \frac{\lambda^{2}}{1-\lambda^{2}} \int \exp \left[-\frac{\left(\lambda q-q^{\prime \prime}\right)^{2}}{1-\lambda^{2}}-\frac{\left(\lambda p-p^{\prime \prime}\right)^{2}}{1-\lambda^{2}}\right] W\left(q^{\prime \prime}, p^{\prime \prime}\right) d q^{\prime \prime} d p^{\prime \prime},
$$

который определяет интегральную связь между функцией Вигнера $W(q, p)$, отвечающей функции Хусими $Q(q, p)$, и функцией Вигнера $W^{\lambda}(q, p)$, соответствующей преобразованной функции Хусими $Q_{\lambda}(q, p)=\lambda^{2} Q(\lambda q, \lambda p)$.

Выясним теперь, что происходит с томограммами при масштабном преобразовании (13). Как мы уже установили, томограммы выражаются через фунции Хусими с помощью формулы (12). Будем считать, что в этой формуле стоят уже преобразованные величины, и запишем ее в виде

$$
w^{\lambda}(x, \mu, \nu)=\frac{1}{(2 \pi)^{2}} \int \exp \left[\frac{k^{2}}{4}\left(\mu^{2}+\nu^{2}\right)\right] Q_{\lambda}(q, p) e^{-i k(x-\mu q-\nu p)} d k d q d p .
$$

4 Теоретическая и математическая физика, т. 166, № 3, 2011 г. 
Подставляя соотношение (13) для преобразованной функции Хусими и используя формулу (11), можно выразить преобразованную томограмму $w^{\lambda}$ через исходную томограмму $w$ :

$$
\begin{aligned}
w^{\lambda}(X, \mu, \nu)= & \frac{\lambda^{2}}{2 \pi} \int w\left(Y, \frac{k \mu}{\lambda}, \frac{k \nu}{\lambda}\right) \times \\
& \times \exp \left[\frac{\mu^{2}}{4}\left(1-\frac{1}{\lambda^{2}}\right)+\frac{\mu^{2}}{4}\left(1-\frac{1}{\lambda^{2}}\right)\right] e^{i(Y-k x)} d Y d k .
\end{aligned}
$$

\section{6. ГАРМОНИЧЕСКИЙ ОСЦИЛЛЯТОР}

В предыдущих разделах мы изучили связи между функциями Вигнера, Хусими и томограммами и выяснили, как эти функции изменяются при масштабном преобразовании (13). Теперь мы хотим разобраться в том, что происходит с физическими состояниями при таком преобразовании. Как уже отмечалось, такая проблема возникает при рассмотрении квантового усилителя [30]. Данную задачу можно решить, воспользовавшись выражениями $(7),(8)$ для ядра оператора плотности $\rho(x, y)$, отвечающего преобразованной функции Хусими. Другой способ предоставляет нам процедура доказательства того, что преобразование (13) переводит функцию Хусими в функцию Хусими (см. приложение). Однако из получающихся таким образом выражений для оператора плотности не всегда можно сразу установить, как устроено физическое состояние, которому он соответствует, например можно легко понять, является ли оно чистым, или смешанным, но узнать, из каких чистых состояний состоит такое смешанное состояние, уже гораздо труднее. Тем не менее в некоторых случаях данную задачу удается решить. В качестве примера рассмотрим гармонический осциллятор, для которого можно установить, как преобразуются его состояния при масштабных преобразованиях (13).

Введем оператор специального вида:

$$
\hat{\rho}_{N}=\frac{\lambda^{2 N+2}}{N !} \sum_{k=0}^{\infty} \frac{(N+k) !}{k !}\left(1-\lambda^{2}\right)^{k}|N+k\rangle\langle N+k|, \quad \lambda^{2}<1 .
$$

ПРЕДЛОЖЕНИЕ. Оператор $\hat{\rho}_{N}$ является оператором плотности.

ДокАзАТЕЛЬство. Из определения (16) непосредственно следует, что оператор $\hat{\rho}_{N}$ является эрмитовым и положительно определенным. Для того чтобы он был оператором плотности, необходимо, чтобы его след был равен единице. Имеем

$$
\operatorname{Tr}\left(\hat{\rho}_{N}\right)=\frac{\lambda^{2 N+2}}{N !} \sum_{k=0}^{\infty} \frac{(N+k) !}{k !}\left(1-\lambda^{2}\right)^{k} .
$$

Поскольку

$$
\sum_{k=0}^{\infty} \frac{(N+k) !}{k !} x^{k}=\frac{N !}{(1-x)^{N+1}}, \quad|x|<1,
$$

получаем, что

$$
\operatorname{Tr}\left(\hat{\rho}_{N}\right)=\frac{\lambda^{2 N+2}}{N !} \frac{N !}{\lambda^{2 N+2}}=1 .
$$

Таким образом, след оператора (16) равен единице, и он является оператором плотности некоторого физического состояния. Предложение доказано. 
Ясно, что оператор (16) описывает смешанное состояние, в которое входят чистые состояния $|N+k\rangle, k=0,1,2, \ldots$, причем каждое из чистых состояний $|N+k\rangle$ входит в смешанное с вероятностью

$$
c_{k}^{N}=\frac{\lambda^{2 N+2}(N+k) !}{N ! k !}\left(1-\lambda^{2}\right)^{k} .
$$

Вычислим теперь функцию Хусими состояния (16):

$$
\begin{aligned}
Q_{\rho_{N}}(q, p) & =\left\langle\alpha\left|\hat{\rho}_{N}\right| \alpha\right\rangle=\frac{\lambda^{2 N+2}}{N !} \sum_{k=0}^{\infty} \frac{(N+k) !}{k !}\left(1-\lambda^{2}\right)^{k} \frac{|\alpha|^{2(N+k)}}{(N+k) !} e^{-|\alpha|^{2}}= \\
& =\frac{\lambda^{2 N+2}}{N !}|\alpha|^{2 N} e^{-|\alpha|^{2}} \sum_{k=0}^{\infty} \frac{\left(1-\lambda^{2}\right)^{k}}{k !}|\alpha|^{2 k}=\frac{\lambda^{2 N+2}}{N !}|\alpha|^{2 N} e^{-|\alpha|^{2}+\left(1-\lambda^{2}\right)|\alpha|^{2}}
\end{aligned}
$$

Окончательно получаем для функции Хусими состояния $\hat{\rho}_{N}$ следующую формулу:

$$
Q_{\rho_{N}}(q, p)=\frac{\lambda^{2}}{N !} \lambda^{2 N}|\alpha|^{2 N} e^{-\lambda^{2}|\alpha|^{2}},
$$

где мы использовали обычное выражение для когерентного состояния $|\alpha\rangle$,

$$
|\alpha\rangle=e^{-|\alpha|^{2} / 2} \sum_{n=0}^{\infty} \frac{\alpha^{n}}{\sqrt{n !}}|n\rangle .
$$

Вычислим теперь функцию Хусими чистого фоковского состояния $|N\rangle$,

$$
Q_{N}(q, p)=\langle\alpha \mid N\rangle\langle N \mid \alpha\rangle=\frac{1}{N !}|\alpha|^{2 N} e^{-|\alpha|^{2}},
$$

и применим к ней масштабное преобразование (13), получим

$$
Q_{N}^{\lambda}(q, p)=\lambda^{2} Q_{N}(\lambda q, \lambda p)=\frac{\lambda^{2}}{N !} \lambda^{2 N}|\alpha|^{2 N} e^{-\lambda^{2}|\alpha|^{2}}
$$

Сравнивая получившееся выражение с (18), мы видим, что они совпадают. Отсюда следует, что при масштабном преобразовании (13) чистое фоковское состояние $|N\rangle$ переходит в смешанное состояние, задаваемое оператором плотности $\hat{\rho}_{N}$.

Рассмотрим эту же задачу с другой стороны. Функция Хусими основного состояния записывается как $Q_{0}(q, p)=e^{-|\alpha|^{2}}$. После масштабного преобразования (13) она приобретает вид

$$
Q_{0}^{\lambda}(q, p)=\lambda^{2} Q_{0}(\lambda q, \lambda p)=\lambda^{2} e^{-\lambda^{2}|\alpha|^{2}}
$$

Ядро оператора плотности, отвечающего преобразованной функции Хусими (19), можно получить, используя формулу (8). Подставляя (19) в (8) и вычисляя интеграл, имеем

$$
\tilde{\rho}_{0}(x, y)=\frac{\lambda^{2}}{\lambda \sqrt{\pi\left(2-\lambda^{2}\right)}} \exp \left[\frac{x^{2}+y^{2}}{2}\right] \exp \left[-\frac{x^{2}+y^{2}}{2-\lambda^{2}}\right] \exp \left[\frac{2\left(1-\lambda^{2}\right) x y}{2-\lambda^{2}}\right] .
$$


Мы хотим убедиться в том, что оператор плотности, отвечающий данному ядру, совпадает с оператором плотности $\hat{\rho}_{0}$ из $(16)$, который имеет вид

$$
\hat{\rho}_{0}=\lambda^{2} \sum_{n=0}^{\infty}\left(1-\lambda^{2}\right)^{n}|n\rangle\langle n| .
$$

В координатном представлении состояние $\langle x \mid n\rangle$ линейного гармонического осциллятора выражается как

$$
\langle x \mid n\rangle=\Psi_{n}(x)=\frac{1}{\sqrt{\pi^{1 / 2} 2^{n} n !}} e^{-x^{2} / 2} H_{n}(x) .
$$

Для ядра оператора плотности (20) получаем

$$
\rho_{0}(x, y)=\lambda^{2} e^{-\left(x^{2}+y^{2}\right) / 2} \sum_{n=0}^{\infty}\left(1-\lambda^{2}\right)^{n} \frac{1}{\sqrt{\pi} 2^{n} n !} H_{n}(x) H_{n}(y) .
$$

Данное соотношение следует преобразовать, используя известное соотношение для полиномов Эрмита [36],

$$
\sum_{n=0}^{\infty} \frac{H_{n}(x) H_{n}(y)}{2^{n} n !} r^{n}=\frac{1}{\sqrt{1-r^{2}}} \exp \left[\frac{2 x y r-\left(x^{2}+y^{2}\right) r^{2}}{1-r^{2}}\right],
$$

подставляя которое в формулу (21), мы получаем равенство $\tilde{\rho}_{0}(x, y)=\rho_{0}(x, y)$.

Аналогичным образом, используя функцию Хусими фоковских состояний $Q_{N}^{\lambda}$, можно установить, что ядра операторов плотности $\tilde{\rho}_{N}(x, y)$ возбужденных состояний, вычисленные с помощью формулы (8), совпадают с ядрами операторов плотности $\hat{\rho}_{N}(x, y)$ из $(16)$.

\section{7. ЗАКЛЮЧЕНИЕ}

Мы нашли дифференциальные и интегральные соотношения, связывающие функции Хусими, функции Вигнера и симплектические томограммы. Дано новое аналитическое доказательство теоремы об инвариантности совокупности функций Хусими относительно масштабных преобразований. Это утверждение справедливо и для многомерных функций Хусими. Тем самым доказано свойство неотрицательности отображения [37] между исходным и конечным операторами плотности, заданного масштабным преобразованием фукции Хусими. При этом получено выражение для матрицы плотности преобразованного состояния. Найдена в явном виде матрица плотности преобразованных фоковских состояний гармонического осциллятора.

В дальнейшем мы предполагаем обобщить полученные результаты на функции Глаубера-Сударшана и рассмотреть различные физические приложения, а также обсудить, является ли масштабное преобразование функции Хусими полностью положительным отображением.

\section{ПРИЛОЖЕНИЕ}

Докажем, что совокупность функций Хусими инвариантна относительно масштабного преобразования (1). Точнее говоря, докажем, что если $Q(q, p)$ - функция Хусими, то $Q_{\lambda}(q, p)$ тоже является функцией Хусими некоторого физического состояния при условии, что $\lambda^{2} \leqslant 1$. 
Для произвольной матрицы плотности $\hat{\rho}$ функция Хусими определена с помощью соотношения (2), где когерентное состояние $|\alpha\rangle$ в $x$-представлении имеет вид

$$
\langle x \mid \alpha\rangle=\frac{1}{\pi^{1 / 4}} \exp \left[-\frac{1}{2}(x-q)^{2}+i p x-\frac{i}{2} q p\right], \quad \sqrt{\alpha}=\frac{q+i p}{\sqrt{2}} .
$$

С помощью равенств (2) и (22) функцию (13) можно представить в виде

$$
Q_{\lambda}(q, p)=\frac{\lambda^{2}}{\pi \sqrt{\pi}} \int \rho(x, y) \exp \left[-\frac{1}{2}(x-\lambda q)^{2}-\frac{1}{2}(y-\lambda q)^{2}+i \lambda p(x-y)\right] d x d y
$$

Для того чтобы доказать, что $Q_{\lambda}(q, p)$ - функция Хусими, необходимо установить два факта. Во-первых, следует показать, что выражение может быть представлено в форме (2), т.е.

$$
Q_{\lambda}(q, p)=\frac{1}{\pi} \int\langle\alpha \mid x\rangle \rho_{\lambda}(x, y)\langle y \mid \alpha\rangle d x d y
$$

Во-вторых, после этого нужно доказать что $\rho_{\lambda}(x, y)$ в правой части этого равенства является матрицей плотности некоторого физического состояния.

Докажем первое утверждение. Для этого рассмотрим тождество

$$
(x-\lambda q)^{2}=(\lambda x-q)^{2}+x^{2}\left(1-\lambda^{2}\right)-q^{2}\left(1-\lambda^{2}\right) .
$$

С его помощью функцию (23) можно представить в виде

$$
\begin{aligned}
Q_{\lambda}(q, p)= & \frac{\lambda^{2}}{\pi \sqrt{\pi}} \int \rho(x, y) \exp \left[-\frac{1}{2}(\lambda x-q)^{2}-\frac{1}{2}(\lambda y-q)^{2}+i \lambda p(x-y)\right] \times \\
& \times \exp \left[-\frac{1}{2}\left(1-\lambda^{2}\right)\left(x^{2}+y^{2}\right)+\left(1-\lambda^{2}\right) q^{2}\right] d x d y
\end{aligned}
$$

Делая замену переменных $\lambda x=u, \lambda y=v$, получаем

$$
\begin{aligned}
Q_{\lambda}(q, p)= & \frac{1}{\pi \sqrt{\pi}} \int \rho\left(\frac{u}{\lambda}, \frac{v}{\lambda}\right) \exp \left[-\frac{1}{2}(u-q)^{2}-\frac{1}{2}(v-q)^{2}+i p(u-v)\right] \times \\
& \times \exp \left[-\left(1-\lambda^{2}\right) \frac{u^{2}+v^{2}}{2 \lambda^{2}}+\left(1-\lambda^{2}\right) q^{2}\right] d u d v
\end{aligned}
$$

Данное выражение не имеет вида (2), поскольку содержит дополнительный множитель $e^{\left(1-\lambda^{2}\right) q^{2}}$. Чтобы устранить это обстоятельство, мы представим этот множитель как результат дифференцирования по переменным $u$ и $v$.

Произведение двух экспонент в правой части (25) можно преобразовать следующим образом:

$$
\begin{aligned}
\exp [- & \left.\frac{1}{2}(u-q)^{2}-\frac{1}{2}(v-q)^{2}+\frac{i p(u-v)}{\hbar}\right] \times \\
& \times \exp \left[-\left(1-\lambda^{2}\right) \frac{u^{2}+v^{2}}{2 \lambda^{2}}+\left(1-\lambda^{2}\right) q^{2}\right]= \\
= & \exp \left[-\frac{u^{2}+v^{2}}{2 \lambda^{2}}-q^{2}\right] e^{\left(1-\lambda^{2}\right) q^{2}} \exp [u(q+i p)+v(q-i p)] .
\end{aligned}
$$


Поскольку справедливо равенство

$$
\begin{aligned}
& e^{\left(1-\lambda^{2}\right) q^{2}} \exp [u(q+i p)+v(q-i p)]= \\
& =\exp \left[\frac{1-\lambda^{2}}{4}\left(\frac{d}{d u}+\frac{d}{d v}\right)^{2}\right] \exp [u(q+i p)+v(q-i p)],
\end{aligned}
$$

мы можем получить для $Q_{\lambda}(q, p)$ выражение

$$
\begin{aligned}
Q_{\lambda}(q, p)= & \frac{1}{\pi \sqrt{\pi}} \int \rho\left(\frac{u}{\lambda}, \frac{v}{\lambda}\right) \exp \left[-\frac{u^{2}+v^{2}}{2 \lambda^{2}}\right] \exp \left[\left(1-\lambda^{2}\right) \frac{1}{4}\left(\frac{d}{d u}+\frac{d}{d v}\right)^{2}\right] \times \\
& \times \exp [u(q+i p)+v(q-i p)] e^{-q^{2}} d u d v .
\end{aligned}
$$

Интегрируя по частям и используя эрмитовость экспоненциальных операторов дифференцирования, получаем

$$
\begin{aligned}
Q_{\lambda}(q, p)= & \frac{1}{\pi \sqrt{\pi}} \int \exp \left[\left(1-\lambda^{2}\right) \frac{1}{4}\left(\frac{d}{d u}+\frac{d}{d v}\right)^{2}\right] \rho\left(\frac{u}{\lambda}, \frac{v}{\lambda}\right) \exp \left[-\frac{u^{2}+v^{2}}{2 \lambda^{2}}\right] \times \\
& \times \exp [u(q+i p)+v(q-i p)] e^{-q^{2}} d u d v
\end{aligned}
$$

Умножая последний сомножитель подынтегрального выражения на $e^{-\left(u^{2}+v^{2}\right) / 2}$ и деля первый сомножитель на такую же величину, получаем произведение двух когерентных состояний $\langle\alpha \mid u\rangle\langle v \mid \alpha\rangle$. Отсюда следует, что выражение (26) можно представить в виде (24).

Таким образом, мы доказали первое утверждение. При этом данное доказательство позволило нам одновременно найти в явной форме и ядро преобразованного оператора плотности $\rho_{\lambda}$ :

$$
\begin{aligned}
\rho_{\lambda}(u, v)= & \exp \left[-\frac{u^{2}+v^{2}}{2}\right] \exp \left[\left(1-\lambda^{2}\right) \frac{1}{4}\left(\frac{d}{d u}+\frac{d}{d v}\right)^{2}\right] \times \\
& \times \rho\left(\frac{u}{\lambda}, \frac{v}{\lambda}\right) \exp \left[-\frac{u^{2}+v^{2}}{2 \lambda^{2}}\right] .
\end{aligned}
$$

Переходим к доказательству второго утверждения. Мы должны показать, что оператор $\hat{\rho}_{\lambda}$, отвечающий ядру $(27)$, является оператором плотности некоторого физического состояния. Для этого необходимо и достаточно, чтобы ядро было положительным и его след равнялся единице.

Вычислим след ядра $\rho_{\lambda}$ :

$$
\operatorname{Tr}\left(\rho_{\lambda}\right)=\int \rho_{\lambda}(u, v) \delta(u-v) d u d v .
$$

Представим $\delta$-функцию в виде

$$
\delta(u-v)=\frac{1}{2 \pi} \int\langle u \mid \alpha\rangle\langle\alpha \mid v\rangle d p d q, \quad \rho_{\lambda}=\int \rho(u, v) \delta(u-v) d u d v,
$$

откуда, принимая во внимание формулы (13) и (24), получим

$$
\int \rho_{\lambda}(u, v) \delta(u-v) d u d v=\int Q_{\lambda}(q, p) d q d p=\int \lambda^{2} Q(\lambda q, \lambda p) d q d p=1 .
$$


Мы доказали, что след функции $\rho_{\lambda}$ равен единице. Теперь нам осталось доказать только то, что она является положительной.

Исходное ядро оператора плотности $\hat{\rho}$ может быть представлено в виде

$$
\rho(x, y)=\sum c_{n} \Psi_{n}(x) \Psi_{n}(y)^{*} .
$$

Все операторы, входящие в выражение (27), коммутируют друг с другом и

$$
\left(\frac{d}{d u}+\frac{d}{d v}\right)^{2}=\frac{d^{2}}{d u^{2}}+2 \frac{d}{d u} \frac{d}{d v}+\frac{d^{2}}{d v^{2}},
$$

поэтому экспоненциальные операторы с двойным дифференцированием по одной переменной не меняют структуру выражения (28), которая обеспечивает его положительность. Действительно, эти операторы действуют только на функции $\Psi$, которые преобразуются в другие функции, но в результате мы опять получаем сумму функций, умноженных на свои комплексно-сопряженные. Если $\left(1-\lambda^{2}\right) \geqslant 0$, то оператор $\exp \left[(1-\lambda)^{2} d / d u \cdot d / d v\right]$ также сохраняет эту структуру.

Таким образом, мы установили, что $\rho_{\lambda}$ является положительной функцией со следом, равным единице. Отсюда следует, что преобразование (13) переводит функцию Хусими в функцию Хусими. Это доказательство можно легко обобщить и на многомерные функции Хусими.

Благодарности. Работа выполнена в рамках научного сотрудничества между Российской Академией наук и Сербской Академией наук и искусств по теме "Фундаментальные исследования в области квантовой теории информации и квантовых вычислений и их приложения". В.А.Андреев благодарит за гостеприимство Institute of Nuclear Sciences Vinca, a Д. М. Давидович благодарен за гостеприимство Физическому институту им. П. Н. Лебедева РАН. В. А. Андреев и Д. М. Давидович благодарят Министерство науки и технологического развития Сербии за поддержку данного проекта. Авторы благодарны РФФИ (грант № 08-02-00741 (В.А. Андреев), грант № 09-02-00142 (В. И. Манько), грант № 10-02-00312 (М. А. Манько)) за частичную поддержку настоящей работы.

\section{Список литературы}

[1] E. Wigner, Phys. Rev., 40:5 (1932), 749-759.

[2] В. И. Татарский, УФН, 139:4 (1983), 587-619.

[3] В. В. Додонов, В.И. Манько, Инварианты и эволючия нестационарных квантовых систем, Труды ФИАН, 183, ред. М. А. Марков, Наука, М., 1987.

[4] K. Husimi, Proc. Phys.-Math. Soc. Japan, 22 (1940), 264-314.

[5] Y. J. Kano, J. Math. Phys., 6:12 (1965), 1913-1915.

[6] R. J. Glauber, Phys. Rev. Lett., 10:3 (1963), 84-86.

[7] E. C. G. Sudarshan, Phys. Rev. Lett., 10:7 (1963), 277-279.

[8] Р. Л. Стратонович, ЖЭТФ, 31:6 (1956), 1012-1020; Докл. АН СССР, 109:1 (1956), $72-75$.

[9] А.С. Холево, Вероятностные и статистические аспекты квантовой теории, ИКИ, Москва, Ижевск, 2003.

[10] Н.Н.Боголюбов (мл.), А. С. Шумовский, "Коллективные квантовые эффекты в задаче о взаимодействии электромагнитного излучения со средой”, Статистическая механика и теория динамических систем, K 80-летию со дня рождения академика Николая Николаевича Боголюбова, Тр. МИАН СССР, 191, Наука, М., 1989, 79-88. 
[11] W. Pauli, Encyclopedia of Physics, v. 5, eds. S. Flügge, Springer, Berlin, 1958, 17.

[12] S. Mancini, V. I. Man'ko, P. Tombesi, Quantum Semiclass. Opt., 7:4 (1995), 615-623; Phys. Lett. A, 213:1-2 (1996), 1-6, arXiv: quant-ph/9603002; Found. Phys., 27:6 (1997), 801-824, arXiv: quant-ph/9609026.

[13] A. Ibort, V. I. Man'ko, G. Marmo, A. Simoni, F. Ventriglia, Phys. Scr., 79:6 (2009), 065013, 29 pp., arXiv: 0904.4439.

[14] D. T. Smithey, M. Beck, M. G. Raymer, A. Faridani, Phys. Rev. Lett., 70:9 (1993), 1244-1247.

[15] П. А. Белоусов, Р. С. Исмагилов, ТМФ, 157:1 (2008), 3-7.

[16] R. F. O'Connell, E. P. Wigner, Phys. Lett. A, 83:4 (1981), 145-148.

[17] M. Hillery, R. F. O'Connell, M. O. Scully, E. P. Wigner, Phys. Rep., 106:3 (1984), 121-167.

[18] K. Takahashi, N. Saitō, Phys. Rev. Lett., 55:7 (1985), 645-648.

[19] D. Biswas, S. Sinha, Phys. Rev. E, 60:1 (1999), 408-415, arXiv: chao-dyn/9904047.

[20] F. J. Arranz, F. Borondo, R. M. Benito, Phys. Rev. E, 54:3 (1996), 2458-2464; J. Chem. Phys., 120:14 (2004), 6516-6523.

[21] H. J. Korsch, C. Müller, H. Wiescher, J. Phys. A, 30:20 (1997), L677-L684.

[22] D. M. Appleby, J. Modern Opt., 46:5 (1999), 825-841, arXiv: quant-ph/9805054.

[23] P. Roy, Optics Comm., 221:1-3 (2003), 145-152.

[24] Э. А. Ахундова, В. В. Додонов, В. И. Манько, ТМФ, 60:3 (1984), 413-422.

[25] H.-Yi. Fan, Q. Guo, Phys. Lett. A, 358:3 (2006), 203-210.

[26] H.-Yi. Fan, Q. Guo, Ann. Physics, 232:6 (2008), 1502-1528.

[27] L. Shunlong, J. Stat. Phys., 102:5-6 (2001), 1417-1428.

[28] F. Olivares, F. Pennini, G. L. Ferri, A. Plastino, Braz. J. Phys., 39:2a (2009), 503-506.

[29] D. M. Davidović, D. Lalović, A. R. Tančić, J. Phys. A, 27:24 (1994), 8247-8250.

[30] G. S. Agarwal, K. Tara, Phys. Rev. A, 47:4 (1993), 3160-3166.

[31] D. M. Davidović, D. Lalović, J. Phys. A, 26:19 (1993), 5099-5105.

[32] K. E. Cahill, R. J. Glauber, Phys. Rev., 177:5 (1969), 1857-1881; 1882-1902.

[33] G. Agarwal, E. Wolf, Phys. Rev. D, 2:10 (1970), 2161-2186.

[34] S. S. Mizrahi, Phys. A, 127:1-2 (1984), 241-264; 135:1 (1986), 237-250; 150:3 (1988), $541-554$.

[35] S. Mancini, O. V. Man'ko, V. I. Man'ko, P. Tombesi, J. Phys. A, 34:16 (2001), 3461-3476, arXiv: quant-ph/0005058.

[36] Г. Бейтмен, А. Эрдейи, Высшие трансцендентные функиии, т. 2: Функиии Бесселя, функиии параболического ицлиндра, ортогональные многочлены, Справочная математическая библиотека, Мир, М., 1974.

[37] E. C. G. Sudarshan, P. M. Mathews, J. Rau, Phys. Rev., 121:3 (1961), 920-924.

Поступила в редакцию 8.06.2010, после доработки 6.10 .2010 